\title{
DÉVELOPPEMENTS LINGUISTIQUES ET APPRENTISSAGE DU VOCABOLAIRE
}

Nous concevons l'apprentissage d'une langue étrangère comme un tout cohérent. Dans cette perspective nous pensons que tous les pôles: grammaire, phonétique et vocabulaire sont complémentaires et d'une importance égale. Or, on a constaté que pour les deux premières beaucoup d'études ont été faites, tandis que les recherches dans le domaine du vocabulaire sont loin d'avoir abouti à des conclusions définitives.

Le souci d'enrichir le lexique des apprenants dans le processus d'apprentissage suppose la conception de différentes stratégies visant l'apprentissage du vocabulaire. Le choix du vocabulaire doit être en fonction du public visé, selon ses besoins, ses objectifs et ses attentes.

Nous verrons à travers cet article comment les développements en didactique et en linguistique peuvent servir à améliorer les techniques d'apprentissage du vocabulaire.

En parlant de l'apprentissage du vocabulaire nous touchons à d'autres domaines, car l'apprentissage ne se fait plus sous la forme d'une liste de mots isolés. Nous avons donc affaire à des réseaux complexes, en particulier, ceux du sens et l'ensemble des relations des différents éléments à apprendre.

Dans cet article notre réflexion sera centralisée en premier lieu sur les questions suivantes:

a) Comment sensibiliser les apprenants arabophones à une meilleure appréhension du vocabulaire français?

b) Comment prendre en considération ce qui caractérise le public arabophone sur le plan linguistique, le milieu d'acquisition, etc.

Notre but vise un apport nouveau dans l'apprentissage du vocabulaire par les arabophones, car nous croyons que des exploitations meilleures sont toujours le résultat d'une réflexion constante sur les causes susceptibles de faire obstacle à,une bonne compréhension du vocabulaire de la langue étrangère. 


\section{La spécificité lexicale}

Comme l'avait déjà constaté A. Martinet "apprendre une langue étrangère, ce n'est pas mettre de nouvelles étiquettes sur des objets connus, mais s' habituer à analyser autrement ce qui fait l'objet de communications linguistiques". ${ }^{1}$ Ajoutons également que "la vocation naturelle des mots est à la polysémie". ${ }^{2} \mathrm{Ce}$ qui aboutit sur le plan interlingual (en l'occurence le français et l'arabe) à des correspondances dissemblables.

Or, nous pensons qu'il est indispensable que les apprenants arabophones prennent conscience, dès le début de leur apprentissage, des dissimilitudes qui existent entre les unités lexicales des deux langues.

Cela devrait nous orienter, en matière de présentation des unités lexicales, vers des procédés appropriés en fonction de spécificités lexicales de chacune de deux langues.

Mais, vu l'étendue de l'ensemble des problèmes relatifs à ce domaine, nous nous limitons à certains aspects importants pour l'apprentissage du vocabulaire par les apprenants arabophones.

\section{Comment apprendre à différencier les mots de sens voisin?}

Afin d'augmenter l'efficacité des procédés d'apprentissage du vocabulaire, il nous semble utile de marquer la différence sémique entre les mots de sens voisin, c'est-à-dire d'expliciter les traits de différence qui devraient permettre d'éclairer les rapports de parenté sémantique entre les mots concernés. Ce qui permettra aux apprenants arabophones de mieux assimiler les mots de sens voisin et, par la suite, de choisir le mot convenable lorsqu' ils utilisent le français dans les différentes situations de communication.

De toute évidence, ce qui intéresse les apprenants est l'utilisation de la langue dans la communication. Il nous semble donc nécessaire de mettre à la disposition des apprenants une description de l'utilisation de la langue.

Prenons, à titre d'exemple, les verbes français: "obliger", "contraindre" et "forcer". Ces trois verbes ont un sens proche, et on peut dire, par exemple:

- Obliger quelqu'un à faire quelque chose,

- ou: forcer quelqu'un à faire quelque chose,

- ou: contraindre quelqu'un à faire quelque chose.

1 Martinet A. 1974, Eléments de linguistique générale, p. 12.

2 Galisson R. 1983, Des mots pour communiquer, p. 5. 
Dans ces phrases, les trois verbes expriment un sens analogue et ils sont ici interchangeables les uns aux autres. Mais cette interchangeabilité est liée au contexte dans lequel les trois verbes sont utilisés.

Un changement de contexte montrera que les trois verbes ne sont pas toujours substituables les uns aux autres. On peut dire par exemple:

- forcer une serrure,

- forcer la porte de quelqu'un

- etc.

Dans ces énoncés le verbe "forcer" ne peut pas être remplacé par le verbe "contraindre" ou "obliger".

Il en est de même pour chacun des verbes "contraindre" et "obliger" qui s'emploient, eux aussi, dans des contextes où il n'est pas toujours possible de remplacer ces verbes l'un par l'autre.

Or, pour prémunir les apprenants arabophones contre le risque d'erreur lorsqu'ils emploient ces verbes, ou d'autres ensembles de verbes de sens voisin, il nous paraît indispensable de fournir un ensemble de contextes contenant le maximum d'informations sémantiques et syntaxiques afférentes au verbe concerné. En particulier, dans les cas où les verbes français ne renvoient pas à un équivalent unique dans la langue maternelle des apprenants.

Le mode de présentation de ces informations (choix des exemples en fonction des particularités lexicales) et la prise en compte des spécificités des apprenants arabophones (niveau de connaissance linguistique, âge, type d'apprentissage antérieur, etc.) peuvent contribuer à un meilleur apprentissage du vocabulaire français.

\section{Comment assimiler les différentes valeurs d'un même mot?}

Les mots possèdent très souvent plusieurs contenus ou plusieurs valeurs. Par conséquent, ils éveillent dans la mémoire de l'apprenant plusieurs significations.

Or, pour épargner aux apprenants arabophones le risque de confusion et afin de faciliter la compréhension de différentes acceptions d'un mot, il nous paraît utile de présenter celles-ci avec les mots qui se trouvent fréquemment dans leur entourage. Par exemple: le verbe français "établir" renvoie à plusieurs significations. Celles-ci sont souvent révélés par les éléments qui entourent le verbe. Les phrases ci-dessous en rendent compte:

- La secrétaire a établi la liste des candidats.

- Le premier ministre a établi un gouvernement.

- La culpabilité de l'accusé est établie. 
Dans ces énoncés le verbe "établir" a respectivement le sens de: "dresser", "constituer" et "prouver".

Lors du passage à l'arabe (la langue maternelle des apprenants), le même verbe français "établir" aura respectivement les équivalents suivants: "wada'a", "šakkala" et "atbata".

Il s'avère donc nécessaire de rendre compte des diverses distributions de chaque valeur d'emploi du mot concerné par le biais de contextualisation.

\section{Propositions}

Comme nous l'avons déjà mentionné, les apprenants arabophones apprennent la langue française en dehors du contexte français. De ce fait, nous pensons qu'il est indispensable de diversifier les activités linguistiques et les exercices portant sur les domaines les plus variés, de façon à permettre à l'apprenant arabophone de communiquer en français avec les moyens linguistiques qu'il est en train d'apprendre.

Dans cette perspective, il convient d'entraîner les apprenants à participer aux diverses techniques d'apprentissage du vocabulaire (conversation, compréhension des documents oraux ou écrits, communiquer en français oralement ou par écrit, etc.). Ce faisant, on permettra aux apprenants non seulement l'acquisition de formes et de structures mais également on entamme la mise en oeuvre de stratégies cognitives.

Enfin la thématique, le rythme d'apprentissage, les techniques d'analyse devraient favoriser chez les apprenants des stratégies de découverte, afin de les inciter à la communication naturelle et les aider à mâtriser progressivement le fonctionnement de la langue française. Ainsi, l'apprenant enrichira lui même son lexique par ses propres moyens et deviendra, par la suite, plus autonome dans son apprentissage.

Les procédés d'apprentissage mis en oeuvre devraient, en effet favoriser la réflexion des apprenants sur le fonctionnement de la langue étrangère, car nous pensons effectivement avec R. Galisson que "les vocabulaires s'apprennent bien davantage qu'ils ne s'enseignent" 3 .

\section{BIBLIOGRAPHIE}

BESSE, H., 1985, Méthodes et pratiques des manuels de langue. Didier, Paris, $184 \mathrm{p}$. BRODIN, E., GOULIER, F., 1988, Une stratégie pour la compréhension orale: Echolangues. Le français dans le monde. Recherches et applications, 145-148. 
CHEVALIER, J.C., 1987, Grammaire et enseignement des langues. Le français dans le monde, recherches et applications, 34-41.

COLIN, J.P., 1972, Dictionnaire des difficultés du français, France.

COSTE, D., 1976, Un niveau-seuil. Conseil de l'Europe (projet langues vivantes), Hatier, Paris, $663 \mathrm{p}$.

DARBELENT, J., 1988, L'apport de la stylistique comparée à L'enseignement de la traduction Meta, Vol. 33, No. 2, 133-141.

DELISLE, J., 1980, L'analyse du discours comme méthode de traduction. University of Ottawa Press, Canada, 282 p.

EDWARDS, J., 1991, Literarcy and education in context of cultural and linguistic heterogeneity. La revue canadienne des langues vivantes, Vol. 47, No. 5, 933-949.

EL HANNACH, M., 1992, Le dictionnaire électronique de l'arabe. Linguistica communicatio. Maroc, Vol. 4, No. I, 81-108.

GAK, V.G., 1977, "Lexicologie contrastive", relations internationales, Mosco, 103-115.

GALISSON, R., 1983, Des mots pour communiquer. CLE international, France, 160 p.

GIACOBBE, J., 1990, Le recours à la langue première, une approche cognitive. Le français dans le monde, Recherches et applications. 115-123.

HARTLEY, B., 1986, Age in second language acquisition. Multilingual Matters, England, 144 p.

HOLEC, H., 1981, A propos de l'autonomie: quelques éléments de réflexion. Etudes de linguistique appliquée. Didier Erudition, 7-24.

MARTINET, A., 1974, Eléments de linguistique générale, Armand Colin. Paris, 221 p.

MONTEIL, V., 1960, L'arabe moderne. Klincksieck, France, 386.

PEYTARD, J., GENOUVRIER, E., 1970, Linguistique et enseignement du français. Larousse, $285 \mathrm{p}$.

PORCHER, L., 1977, Pour une sociologie des apprentissagès. Le français dans le monde, No. 133, 36-41.

RAMAT, P., 1985, Typologie linguistique. P.U.F. France, 139 p.

REHAIL, H., 1992, Equivalence, culture et traduction. Linguistica, Ljubljana, Vol. 32, 221-225.

ROMAN, A., 1992, Système et structure en langue. Linguistica communicatio, Maroc, Vol. 4, No. I, 10-23.

Povzetek

JEZIKOSLOVNI PRISTOPI PRI USVAJANJU BESEDIŠČA

Prispevek opozarja na pomembnost obvladovanja besedišča pri usvajanju tujega jezika. Sodobne jezikoslovne študije dokazujejo, da imamo pri usvajanju besedišča $v$ tujem jeziku opraviti z zapletenimi povezanostmi: te zadevajo dojemanje pomena in pa povezanosti med besednimi prvinami, ki jih skušamo usvojiti. Arabsko govoreči naj bi pri učenju francoskega jezika z natančnim razčlenjenjem pomenskega polja obogatili svoje besedišče. 